\title{
Body Mass Index reference curves for Sri Lanka
}

\author{
P M G Punchihewa ${ }^{1}$, L P C Saman Kumara ${ }^{2}$ \\ Sri Lanka Journal of Child Health, 2001; 30: 98-102
}

(Key words: Body mass index, reference curves, Sri Lanka)

\begin{abstract}
Body Mass Index (BMI) or weight/height ${ }^{1,2}$ has been popular for assessing obesity in adults for many years, but its use in children has developed only recently. B.M.I. is widely accepted, as it provides a convenient measure of obesity, which is less affected by differences in timing of puberty than weight for height charts. Child BMI age reference charts have been published in several countries and the aim of this paper is to formulate a set of curves for Sri Lankan children
\end{abstract}

\section{Method}

1182 school children from year 01 to year 07 from Galle District were included in this study group. Standing height was measured to the nearest $0.1 \mathrm{~cm}$. Children were weighed with the least amount of clothes using a calibrated mechanical scale. Children with diagnosed growth disorders and those on medication known to interfere with growth were excluded.

Statistical analysis of data obtained, were entered into a computer and analyzed according to a previously published programme used, for the derivation of general population centiles. The results have been calculated as centiles and as SD scores.

\section{Introduction}

Weight and Height are highly correlated during childhood, so that a child's weight centile tends to be strongly influenced by his or her height centile. Both are a reflection primarily of the child's size rather than their shape. Weight adjusted for height provides a simple measure of fatness and many weight-for-height indices have been proposed for this purpose. The American National Centre for Health Statistics (NCHS) growth reference included weight for height charts. A problem with these charts is that they fail to adjust for age in early life, when body fat content differs substantially ${ }^{3}$. A very flexible and convenient measure of a person's fatness, which removes most of the trend of increasing weight with age, is BMI defined as:

Body weight in $\mathrm{kg}$

Square of the height in metres

${ }^{1}$ Consultant Paediatrician, ${ }^{2}$ Paediatric Registrar,
Teaching Hospital, Karapitiya.
BMI as a measure for underweight and over-weight is used in adults but its use in childhood has developed relatively recently. Adult BMI increases fairly slowly with age, so that age independent cut offs can be used to grade obesity. In children, however BMI changes substantially with age, rising steeply in infancy falling during the pre-school years and then rising into adulthood. For this reason childhood BMI needs to be assessed using age related reference curves. The aim of this pilot study is to explore the possibility of providing reference curves for BMI covering the age range of 0516 years.

\section{Subjects}

The reference sample of children was obtained from 17 schools from Galle district. These schools included National schools as well as others. They were randomly selected from a list of schools obtained from The Ministry of Education of Southern Province. After obtaining prior permission, these schools were visited, and the students from grade 01 to grade 07 were randomly selected making use of the attendance register. Children with diagnosed growth disorders and those on medication known to interfere with growth were excluded. This sample contained a socioeconomic mixture of privileged and less privileged families.

\section{Data}

Trained medical professionals performed measurements. Standing height was measured to the nearest $0.1 \mathrm{~cm}$. Children were weighed with minimum amount of clothes or the weight was adjusted for clothing weight. Exact date of birth was obtained from the attendance register.

\section{Statistical Analysis}

BMI was calculated from the weight and height with units, $\mathrm{kg} / \mathrm{m}^{2}$. The analyses were done for the sexes separately. Since the distribution of BMI in a population tends to be positively skewed, the BMI reference centiles were derived using the LMS Method ${ }^{4}$. In brief this method summarizes the centiles by 03 smooth curves representing the distribution of BMI at each age by its median (M curve) and coefficient of variation ( $\mathrm{S}$ curve) plus a measure of skewness ( $\mathrm{L}$ curve) required to transform the data to normality. The $\mathrm{M}$ curve is the 50th centile curve for BMI. 


\section{Results}

The data set consists of BMI for 1182 children, out of whom 756 were females and 426 were males. Age distribution of the studied population was from 5 to 17 years. The age and sex distribution of the population is shown in Figure 1.

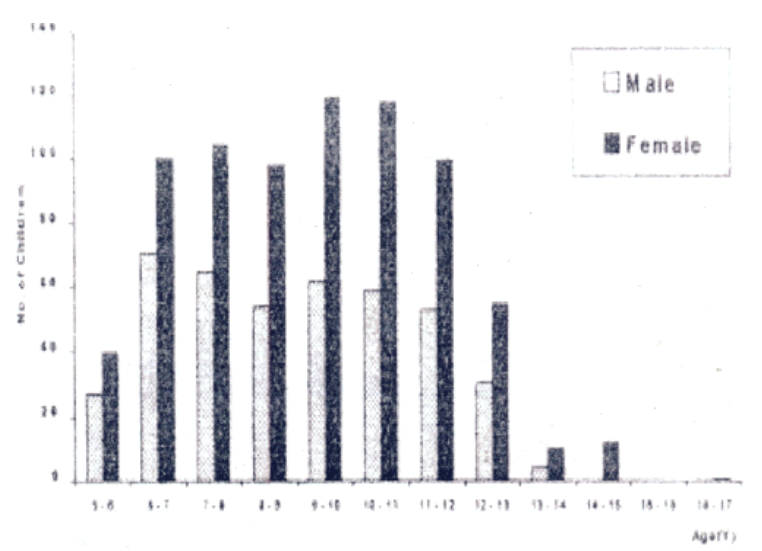

Figure 1 Age and sex distribution

The changes in median BMI by age are on the whole very similar in the 02 sexes. After the adiposity rebound (which is the dip in the BMI) the BMI curve increases more rapidly in girls than in boys. (Figure 2)

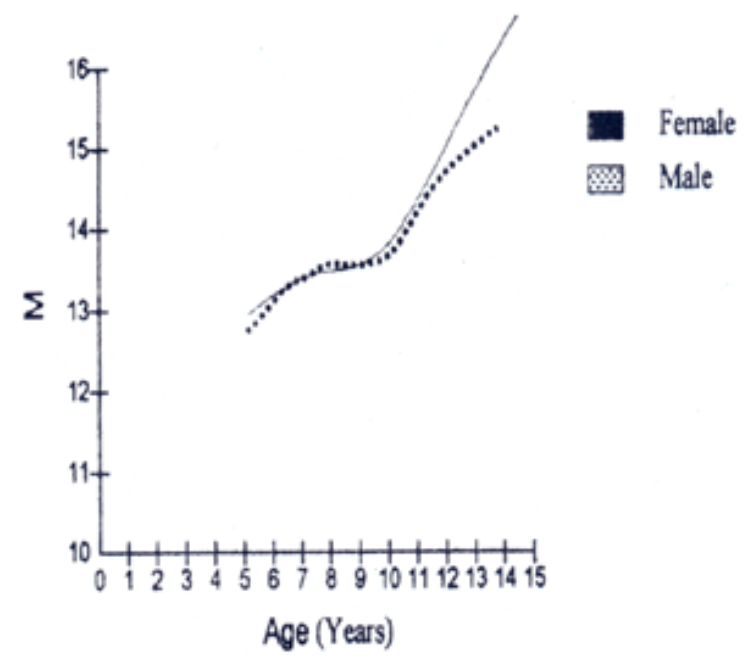

Figure 2 Comparison of male and female median curves

When compared, our findings are consistent with British data, note the rapid increase of BMI in girls after the adiposity rebound; at 18 years the curves cross to give rise to subsequent higher BMI for boys.(Figure 3 )

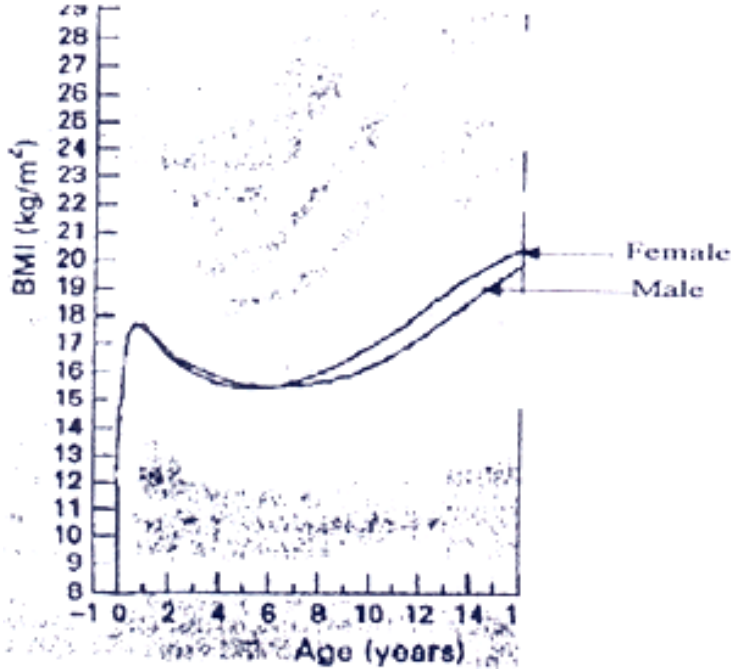

Figure 3 Comparison of British male and female median curves

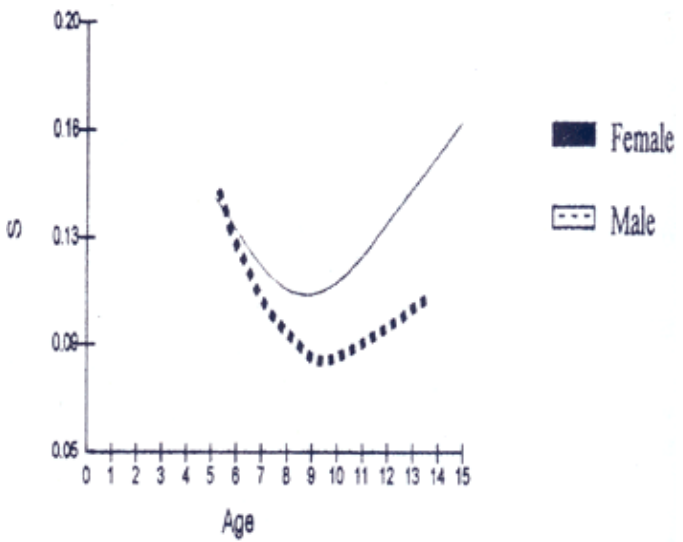

Figure 4 Comparison of coefficient of variation of BMI (S Curve) male and female

Figure 4 shows the $\mathrm{S}$ curves (coefficient of variation of BMI) of boys and girls. The rise in variability occurs earlier in girls reflecting the timing of the adolescent growth spurt in the two sexes.

BMI reference centiles for our population of boys and girls are shown in the seven centile format in Figure 5. Seven centiles are similar to the conventional 3rd, 10th, 25th, 50th, 75th, 90th and 97th.

Figure 6 compares our results with British 50th centile for girls and boys. B.M.I. is consistently higher in the British population. Their 50th centile for females falls on our 90th centile and that of boy's falls well above our $97^{\text {th }}$ centile. 

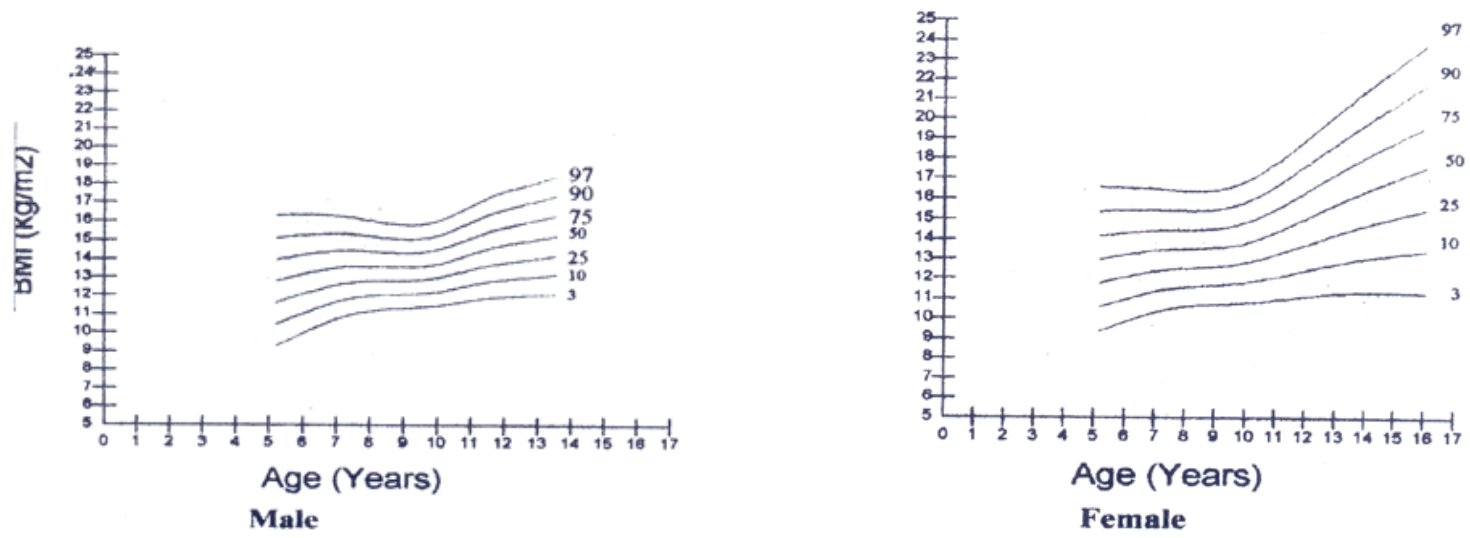

Figure 5 BMI reference curves
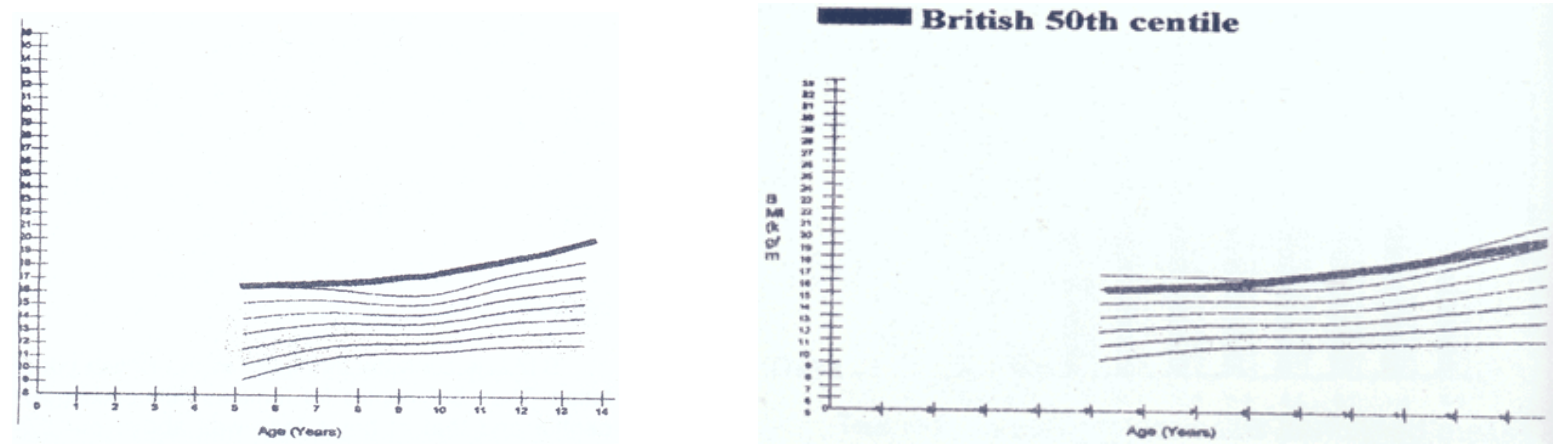

Figure 6 Comparison with British boys and girls

\section{Discussion}

BMI charts can be used to identify children who are unusually fat or thin on the basis of a single measurement and provides an index, which is very useful for large-scale epidemiological purposes. It is less affected by differences in timing of puberty than weight for height charts.

British BMI charts recently published by the Child Growth Foundation spans from birth to 23 years and the centiles were fitted using Cole's LMS method, which is what we used for analysis of our data. In a comprehensive review of more than 60,000 healthy children from the USA, aged 5-17 years, it was shown that Asian children have the lowest values. ${ }^{5}$. This was shown to be the case for adult population as well. Comparison of our results with British BMI is consistent with these findings and this difference is secondary to genetic and environmental impacts on growth. It is clear now that it could create serious anomalies in classification of growth if we continue to use British reference curves. As shown on this British chart, BMI increases steeply in early life, subsequently it declines and then flattens out. This dip - the adiposity re-bound, occurs earlier on the higher than the lower centiles with a difference of up to 3-4 years (Figure 7).
This phenomenon explains why our charts show adiposity rebound as late as 08 years when com-pared to British adiposity rebound of 5.5 years. BMI curves published in 1995 by the Family Health Bureau in Collaboration with Medical Research Institute has taken 5 th and 85th centiles as reference curves. When comparing, our 5th and the 85th centiles are significantly lower than theirs (Figure 8).

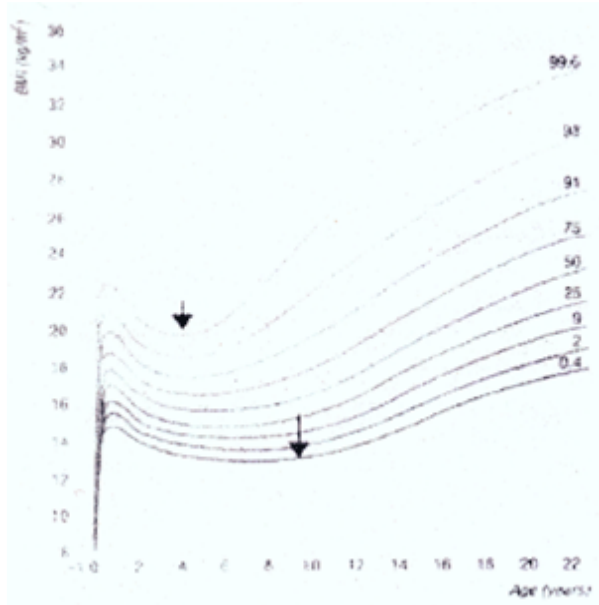

Figure 7 British B.M.I. chart demonstrating adiposity rebound 

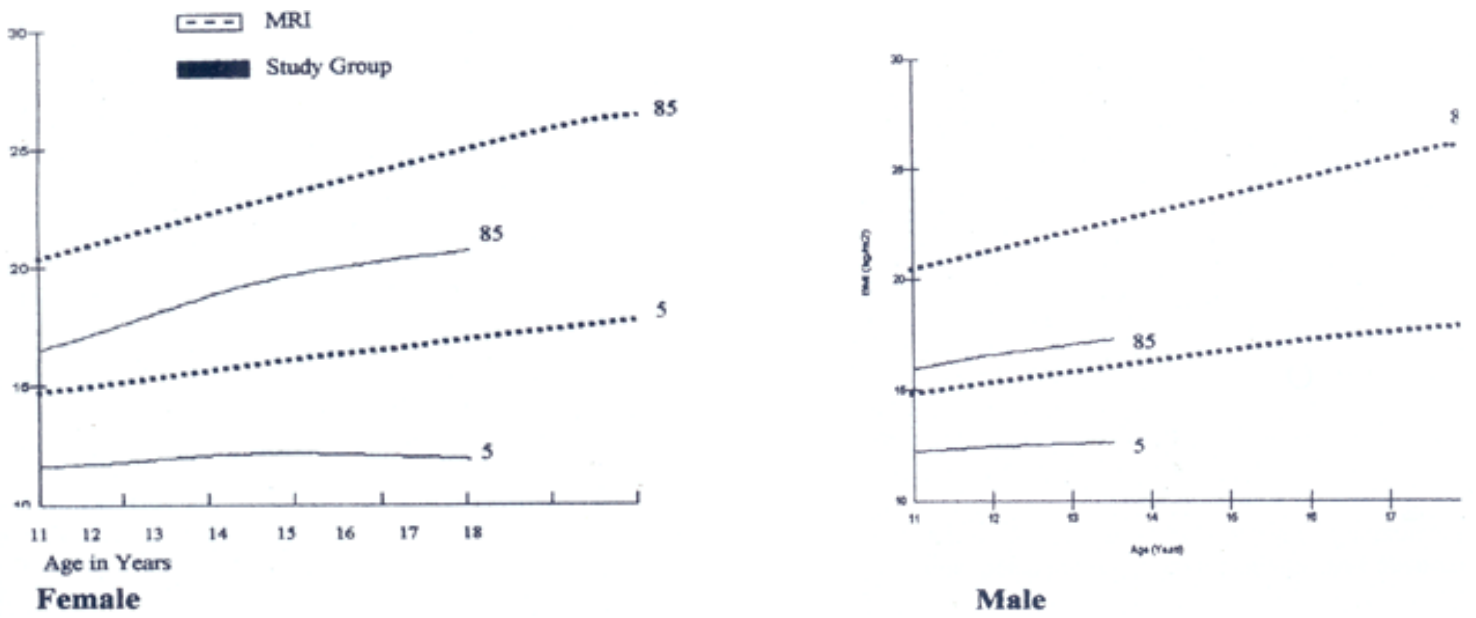

Figure 8 Comparison of 5th and 85th centiles against MRI data. .

Though the sample size is small we emphasize that the urban population we surveyed contains a socioeconomic mixture representative of the national urban pattern.

\section{In conclusion}

- BMI charts taken in conjunction with those for height and weight provide a measure for underweight and overweight.

- These findings signal the need to have our national charts for height, weight and BMI.

- It is important to update growth curves regularly to demonstrate the secular changes

\section{Acknowledgements}

We are ever so grateful to Prof. Tim Cole, Professor of Medical Statistics at the Institute of Child Health, UK for the statistical support and the encouragement given in preparing this paper. We would also like to thank all the principals for granting permission, teachers and all the students who were involved in this study.

\section{References}

1. Prentice A M. Body mass index standards for children. BMJ 1998; 317: 1401-13.

2. Preece M, Cole T, Fry T. Body mass index standards for children. $B M J 319 ; 122 \mathrm{a}-122$.

3. Cole T J. A critique of the NCHS weight for height standard. Hum Biol 1985; 57: 183-96.

4. Cole T J. A method for assessing age-standardized weight-for-height in children seen crosssectionally, Ann Hum Biol 1979; 6: 249-68.

5. Freeman J V, Cole T J, Chinn S, Jones P R M. Cross sectional stature and weight reference curves for the UK, 1990. Arch Dis. Child 1995; 73: 17-24. 
